Erratum

\title{
Erratum to: The raspberry model for protein-like particles: Ellipsoids and confinement in cylindrical pores
}

Vincent D. Ustach and Roland Faller

Department of Chemical Engineering, University of California Davis,

One Shields Ave, Davis, CA 95616, USA

Eur. Phys. J. Special Topics 225, 1643-1662 (2016)

http://dx.doi.org/10.1140/epjst/e2016-60089-7

The supplementary material, in the form of one pdf file, is available from the journal web page at http://dx.doi.org/10.1140/epjst/e2016-02676-9

The Publishers regret this mistake and apologize for any inconvenience caused. 